\title{
Localized Multispectral Crop Imaging Sensors Engineering \& Validation of a Cost Effective Plant Stress and Disease Sensor \\ DOI:
}

10.1109/SAS.2015.7133588

Link to publication record in Manchester Research Explorer

Citation for published version (APA):

Grieve, B., Gurkan, D. (Ed.), \& Baglio, S. (Ed.) (2015). Localized Multispectral Crop Imaging Sensors Engineering \& Validation of a Cost Effective Plant Stress and Disease Sensor. In D. Gurkan, \& S. Baglio (Eds.), IEEE 2015 Sensors Applications Symposium IEEE. https://doi.org/10.1109/SAS.2015.7133588

Published in:

IEEE 2015 Sensors Applications Symposium

\section{Citing this paper}

Please note that where the full-text provided on Manchester Research Explorer is the Author Accepted Manuscript or Proof version this may differ from the final Published version. If citing, it is advised that you check and use the publisher's definitive version.

\section{General rights}

Copyright and moral rights for the publications made accessible in the Research Explorer are retained by the authors and/or other copyright owners and it is a condition of accessing publications that users recognise and abide by the legal requirements associated with these rights.

\section{Takedown policy}

If you believe that this document breaches copyright please refer to the University of Manchester's Takedown Procedures [http://man.ac.uk/04Y6Bo] or contact uml.scholarlycommunications@manchester.ac.uk providing relevant details, so we can investigate your claim.

\section{OPEN ACCESS}




\title{
Localized Multispectral Crop Imaging Sensors
}

\author{
Engineering \& Validation of a Cost Effective Plant Stress and Disease Sensor
}

\author{
Bruce Grieve, Simon Hammersley \\ School of Electrical \& Electronic Engineering \\ The University of Manchester \\ Oxford Road, Manchester, M13 9PL, UK \\ bruce.grieve@manchester.ac.uk
}

\author{
Anne-Katrin Mahlein, Erich-Christian Oerke, \\ Heiner Goldbach \\ Institute for Crop Science and Resource Conservation \\ (INRES) - Plant Nutrition, University of Bonn, \\ Meckenheimer Allee 166a, 53115 Bonn, Germany
}

\begin{abstract}
Close proximity hyperspectral and multispectral imaging of crops and soils offers significant potential to optimize sustainable intensification of arable produce and seeds breeding, through the real-time precision management of plant pathogens, viruses and pests and the non-destructive high throughput screening for beneficial crop traits. These opportunities have been recently reported and are the subject of ongoing $R \& D$ within industry and academia. The broad uptake of the technology by large commercial end-users, through integration with in-field and glasshouse machinery, is limited by cost and equipment reliability. It is further restricted by spectral and spatial resolution, power budget and size, when extending its applicability to consumer markets and small-holder farmers. This study verifies, for the first time, that multispectral sensor systems architectures, exploiting proprietary narrowband LEDs and silicon C-MOS imaging detectors, are capable of substituting for conventional and more expensive line-scanning hyperspectral imaging systems when operated in close proximity (c. 1-2m) of a crop canopy. This was achieved by comparing the data from a prototype version of the new LED-sensor system versus a reference laboratory hyperspectral imaging unit, which was previously developed for crop phenotyping, and the early detection of two fungal pathogen borne diseases in whole barley and sugar beet plants. The choice of crops and diseases replicates earlier studies, with the reference hyperspectral unit, and serves to demonstrate the generic applicability of the new LED-sensor system to cereal and tuber classes of crops. The results indicate that the new approach can deliver data of comparable quality to that of the reference system, for in-field duties, and offers the opportunity for higher sensitivity and spatial resolution. Future potential to apply the new multispectral, LED-based system within commercial products is then discussed.
\end{abstract}

Keywords-hyperspectral, crop, agriculture, disease, fungal pathgen, barley, sugar beet, stress, sensor, instrument

\section{INTRODUCTION}

Remote sensing of crop and terrestrial features through the imaging of analysis of visible (VIS, 400-700 nm), near infrared (NIR, 700-1000 nm) and short wave infra-red (SWIR, 1000$2500 \mathrm{~nm}$ ) wavelengths has been exploited by the agriculture and food supply industry for a number of years [1-3]. Typically the hyperspectral imaging (HIS) sensor units, capable of scanning VIS, NIR and SWIR ranges, have been located on aircraft or orbiting earth-observation satellite platforms and have mapped broad areas of land through calculating Spectral

This work was supported by the UK Engineering and Physical Sciences Research Council (EPSRC) under the Manchester Image Reconstruction and ANalysis (MIRAN) grant, reference: EP/K00428X/1.
Vegetation Indices (SVI) [4], one of the earliest and most common being Normalized Difference Vegetation Index (NDVI) [5]. NDVI utilizes the 'red-edge' which represents the significant difference in reflectance of a crop canopy to light of wavelengths around $700 \mathrm{~nm}$. With reference to (1), $R_{750}$ and $R_{660}$ are the reflected sunlight intensities at $750 \mathrm{~nm}$ (NIR) and $660 \mathrm{~nm}$ (Red) wavelengths, respectively. These reflected wavelengths represent the divide where photon energies are less than sufficient for photosynthesis by chlorophyll $\mathrm{a}$ or $\mathrm{b}$ and so their absorbance by plants would result in heating and potential damage of the tissue by sunlight.

$$
N D V I=\frac{R_{750}-R_{660}}{R_{750}+R_{660}}
$$

NDVI, and subsequent SVIs, use subsets of the HSI data, conventionally ratioing image pixel-intensities at one wavelength with their corresponding values at one or more alternate wavelengths. As a consequence Multispectral Imaging (MSI) camera systems are often subsisted, for full spectrum HSI detectors, to detect specific SVIs. MSI detectors incorporate narrowband light detectors or filter assemblies which are specifically tuned to the required wavelengths for the SVIs. This enables MSI sensors to be manufactured more cost effectively than HSI cameras and potentially to offer more wavelength accuracy and higher scanning speeds. However, the aerial positioning of these large area scanners dictates that they require sunlight energy as the only practicable source of light energy. This has the disadvantage that the reflected light energy available at any given narrowband of wavelengths will be low under normal crop production environments. Thus, to gain suitable signal-to-noise ratio (SNR) at the necessary wavelengths for SVIs requires high-sensitivity detectors which, due to the movement of the aircraft or satellite, must also be capable of capturing the light energy at relatively high speed to achieve adequate spatial resolution. This requires remote HSI and MSI cameras to have high light sensitivity, often incorporating cooling units to reduce the thermal generation of electron transport ('dark current') in the photon detectors. To reduce system cost and payload weight, the resulting detector assemblies are commonly combined with line- and rasterscanning optical beam steering subsystems so that one, or a small number, of detectors can map the required image area.

In more recent years work has been reported on closeproximity, i.e. in-field, analysis of spectral and hyperspectral 
crop features. Research include both abiotic and biotic stress detection, for drought [6], pests [7] and pathogens [8], as well as speciation of crops versus parasitic weeds [9], estimation of soil carbon [10] and wheat protein content [11]. These studies have tended to adopt the remote passive HSI and MSI detectors, from the geospatial imaging sector, and then to take advantage of the variation in plant and soil data arising intra and inter crop canopy to identify new spectral features. This spatial and spectral information, when incorporated into models alongside appropriate meta-data, shows significant potential for HSI and MSI to provide rapid and non-invasive methods of autonomously detecting pre-visual symptoms of specific plant stresses, early emergence of weeds and screening of useful phenotypic traits. However, to achieve broad uptake of these techniques by the commercial agriculture industry requires the current hyperspectral camera's functionality to be deviceengineered into a fundamentally lower cost and robust technology.

When translating HSI to the field environment, there is less of a need to be reliant on sunlight as the sole source of light energy. For example, fluorescence imaging of chlorophyll activity is a commonly used technique which is reliant on the active illumination of leaf samples from dark- and semi-dark adjusted plants [12]. Similarly the laser lighting has been applied to gain spectral data from crop canopies [13] and closed environment Light Emitting Diode (LED) lit transportable chambers have been deployed on tractors for field phenotyping using SVIs [14]. Commercial non-imaging units also exist for inferring nitrogen content, notably the N-Sensor ${ }^{\mathrm{TM}}$ from Yara International ASA (Norway), though these are reliant on passive sensing of the SVI relevant wavelengths [15].

This paper introduces the design and validation of a new sensor architecture that can deliver high spatial-resolution MSI data suitable for close proximity mapping and analysis of plant features. The approach reported has been engineered to meet the needs of commercial crop production, of broad-acre cereal and horticultural crops, as well as field phenotyping for industrial seeds breeding. To achieve this it incorporates a modified proprietary silicon C-MOS (Complementary Metal Oxide Semiconductor) detector array integrated with VIS-NIR optics, synchronized LED active-illumination and low computationally-intensive vision processing to provide a technology platform for direct in-field use within massproduction handheld or machine mounted instrumentation. To demonstrate the effectiveness of the new MSI sensor system, its operation was verified through comparative analysis with a reference laboratory HSI instrument, which was configured to replicate earlier studies on fungal pathogen emergence in sugar beet [16] and barley crops. The latter are representative of monocotyledons and dicotyledonous plants.

\section{Close Proximity Sensor System Engineering}

\section{A. System Concept}

Mass produced silicon ( $\mathrm{Si}$ ) imaging arrays, as embedded within digital cameras and smartphones, are capable of detecting electromagnetic wavelengths in the approximate range $400-1100 \mathrm{~nm}$. The longer wavelength limit is controlled by the energy of the incident photon. As a photon interacts with other particles by transferring its energy to the other particle and then disintegrates itself. This exchange of energy happens if the maximum energy that the photon can bring is at least equal to the smallest quantum of energy that the other particle can accept. For a monocrystalline silicon lattice, this smallest quantum is $1.1 \mathrm{eV}$, referred to as the 'silicon band gap' [17]. From quantum mechanics (2) this equates to a photon having a wavelength of $1125 \mathrm{~nm}$ or shorter. Where: $E_{p h}$ is photon energy $\left(\mathrm{J}, 1 \mathrm{eV}=1.602 \times 10^{-19} \mathrm{~J}\right), h$ is Plank's constant $\left(6.626 \times 10^{-34} \mathrm{Js}\right), \mathrm{c}$ is the speed of light in a vacuum $\left(2.998 \times 10^{8} \mathrm{~m} / \mathrm{s}\right)$ and $\lambda$ is wavelength $(\mathrm{m})$

$$
E_{p h}=\frac{h c}{\lambda}
$$

For photons of wavelengths shorter than $1125 \mathrm{~nm}$ the probability of interaction with silicon is controlled by the absorption coefficient $\alpha\left(\mathrm{m}^{-1}\right)$. From the Beer-Lambert relationship (3), a photon penetration depth can be defined at the thickness of material required to absorb $1 / e(37 \%)$ of the incoming radiation. Where: $I(z)$ is the intensity of photons at depth $z$ into the material, for $I_{o}$ incident light at the surface, and $e$ is Euler's number $(\sim 2.718)$

$$
I(z)=I_{0} e^{\alpha z}
$$

For silicon, at room temperature, this equates to less than a $1 \mu \mathrm{m}$ penetration at below $400 \mathrm{~nm}$ wavelengths [18], see Fig. 1, so dictating a shorter wavelength limit on practical Si C-MOS detectors. Similar logic may be applied to the usable emission wavelengths of Si-LEDs.

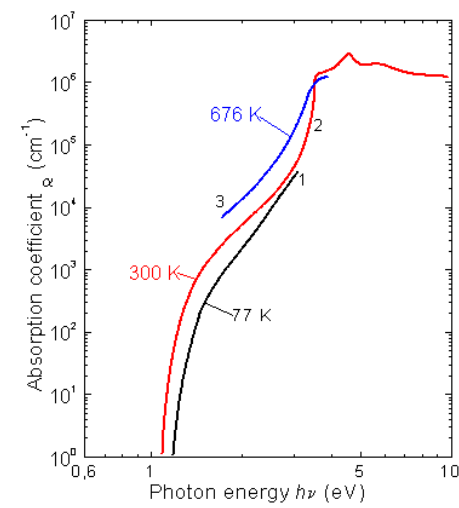

Fig. 1. Silicon photon absorption coefficient vs. photon energy at different temperatures (source 1,2[18] \& 3 [19])

Despite these physical limitations on silicon optical components, it is clear that they are capable operating at wavelengths longer than the visible region and well into the NIR. The reason why consumer digital imaging devices are frequently limited in their wavelength range is through the inclusion of an optical low-pass filter to specifically prevent the interference of non-visible wavelengths within visual images. However, these filters are not an inherent part of C-MOS detectors and are readily removable. Some manufacturers of commodity cameras now adopt the policy of dispensing with the cost of the filter entirely and using software to correct for NIR light interference.

LEDs, whether they generate non-coherent or laser light, are inherently narrow bandwidth. This is by virtue of the wavelength of emission being dictated by the band gap of the 
pure or alloyed semiconductor materials used. Through combining these narrowband light emitters with the broadband C-MOS imaging detectors it is then possible to strobe through a series of SVI relevant wavelengths through sequentially lighting the object under investigation with the corresponding LED light source. Provided the degree of illumination, in each case, does not saturate the detector and the image sequence can be collected at a rate where the object remains constant throughout, or any movement can be compensated for, then it is possible to create a MSI by subtracting a reference ambient lit image from the raw image data-set. This is the basic operating premise behind the new MSI sensor system for in-field crop analysis.

\section{B. Sensor System Engineering for Fungal Pathogen Trial}

The MSI sensor system, used within the fungal pathogen investigation, was comprised of a Canon Model 1100D Digital Single Lens Reflex (D-SLR), which was modified through extraction of the low-pass filter (source: Baadar Planetarium Gmbh, Mammendorf, Germany). This unit was then embedded within a light-board assembly, comprised of over $1000,5 \mathrm{~mm}$ diameter, narrowband LED emitters which were spectrally selected to emit light with center wavelengths at 530, 570, 670, 735 and 830nm (source: Roithner Lasertechnik GmbH, Vienna, Austria). Each LED having a Full Width at Half Maximum (FWHM) of 15-20nm. The specific wavelengths being selected as the most significant bands for the SVIs previously identified from the reference fungal pathogen studies (see Section III, [16]). The numbers of each class of LED being defined by their individual theoretical output efficiencies, such that approximately identical emission intensities were obtained from each lighting-cluster. Fine adjustment of the overall light intensity, at each wavelength, was then adjusted through Pulse Width Modulation (PWM) of individual current sources, which were addressable per wavelength cluster. The PWM period was set to be at least an order of magnitude shorter than the minimum camera exposure time, to ensure uniformity of illumination without the need to accurately synchronize image frame-capture with the current modulation. Specular reflectance was reduced, without the inclusion of a light diffuser element through orientation of the individual LEDs with respect to the parallel camera lens assembly such that their cone of light emission would not result in direct reflection of the light energy into the detector by an orthogonal reflective surface, such as a waxy leaf. The LEDs, making up each light cluster, being evenly distributed over the surface of the composite light board, see Fig 2, to gain even light distribution within the camera's field of view.

The PWM duty cycle could then be adjusted, per wavelength, by software to then compensate for the quantum efficiency of both the imaging detector and the specific LED components. This was achieved by using an optically 'gray' (half-energy) diffuse reflectance tile, suitable for VIS and NIR wavelengths (source: Thorlabs Ltd, Ely, UK) and balancing the total light intensity, at each wavelength, such that a signal equal to approximately half the detectors dynamic range was achieved with the camera aperture set to minimum. The latter was a precaution to increase the focal depth of the optical assembly and so minimize the effects of chromatic aberration, especially at the NIR wavelengths where a proprietary visual camera lens may not be optimized.

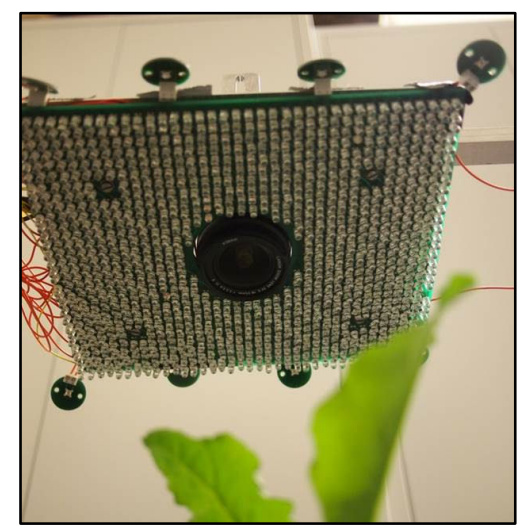

Fig. 2. LED lighting assembly with center wavelengths at 530, 570, 670, 735 and $830 \mathrm{~nm}$ showing location of camera in centre.

Low-level control of the current sources and camera shutter activation was provided through a dedicated embedded microcontroller card integrated with bespoke drive electronics.

\section{VALIDATION OF SENSOR SYSTEM FOR EARY FUNGAL PATHOGEN DETECTION}

\section{A. Equipment and Operating Procedure}

The validation trial, for the new MSI sensor system, was performed on two model systems. The first using pathogen inoculated barley and the second with inoculated sugar beet. Both studies exploited the same reference HSI instrumentation (for detailed description see [20]), located at INRES (University of Bonn), and the new experimental MSI sensor system, as shipped from the University of Manchester. The reference system comprised of two hyperspectral line cameras and associated lighting attached to a linear translation gantry, as shown in Fig 3, positioned approximately 1 meter above crop samples. Only one of the cameras, the ImSpector V10E (source: Spectral Imaging Ltd, Oulu, Finland) with a spectral resolution of $2.8 \mathrm{~nm}$ from $400-1000 \mathrm{~nm}$ and a spatial resolution of $0.19 \mathrm{~mm}$ ( 1650 pixels per line), was used in the study as the spectral range of the second was incompatible with the investigation.

To acquire data from the HSI instrumentation the following procedure was followed, this took approximately 10 minutes per camera for each plant sample: The system was calibrated against a white reference bar, comprised of barium sulfate which extended across the scan line of the cameras; The linear stage was moved across the entire width of the reference bar with a scan speed linked to the frame rate of the hyperspectral camera and to the distance to the object; The previous step was repeated with the shutter of the camera closed to acquire a dark current measurement; The white reference was then removed and the camera shutter re-opened; New start and end points were then programmed for the gantry, which covered the entire range of interest for the specimen plant, followed by recording a scan of the specimen in a similar manner to the calibration scan and; A second dark current reading was taken for the entire image and the data from the white reference and the dark 
current readings were then used to correct for the spectral and spacial variations of the light source and camera sensitivity. This process was undertaken using in-house software. The scanning process and the camera controlling was undertaken using the Data cube software (Spectral Imaging Ltd, Oulu, Finland), the normalization routine and data preprocessing was undertaken using in-house software in ENVI/IDL. The output files from the normalization procedure were then available for analysis in a similar manner to that previously reported [16].

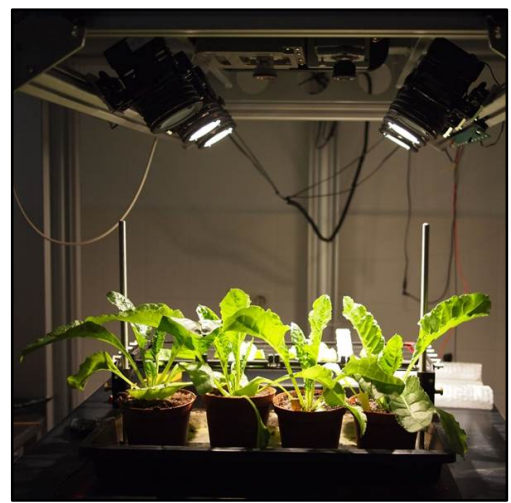

Fig. 3. Reference HSI camera assembly on gantry, showing ImSpector (top centre left) camera and halogen light sources

As the new MSI system captured a complete image, at each of the illumination wavelengths, the camera was positioned centered approximately 1 meter above the plant specimens such that it had a similar field-of-view to the reference line-scanning HSI instrument and was triggered autonomously, via the embedded microcontroller. Image recording followed the procedure as follows: A first dark current reading was taken with no illumination; The object was illuminated with the first SVI wavelength and the image captured after a short delay $(>100 \mathrm{~ms})$ to enable stabilization of the LED lighting cluster, which remained lit for a short period $(>100 \mathrm{~ms})$ after image capture; The object was illuminated and image captured, as before, for the second, and all remaining, SVIs in sequence and; A second dark current reading was taken to enable sensor drift to be retrospectively compensated for. A reference 'gray tile' was included in the MSI sensor system's image space, to enable internal calibration of the data during post-processing of the information. Typically the full cycle took less than a minute for a 3 component SVI. This delay was dominated by the comparatively slow access time of the memory card in the DSLR camera.

\section{B. Experimental Programme for Validation of Sensor System for Early Detection of Fungal Pathogen Crop Diseases}

The HSI and MSI sensor systems were investigated at the example of barley (Hordeum vulgare) and relevant foliar plant diseases caused by fungal pathogens: Powdery mildew (Blumeria graminis f. sp. hordei; Brown rust (Puccinia hordei) and; Net blotch (Pyrenophora teres) This was followed by complementary trials on sugar beet plant samples (Beta vulgaris) and the plant diseases: Cercospora leaf spot (Cercospora beticola)and; sugar beet rust (Uromyces betae)For the purposes of reporting the sensor system validity, only one protocol for pathogen inoculation will be detailed for each plant species followed by the corresponding results from the instruments.

Barley Inoculation Protocol for Powdery Mildew disease: As a biothrophic pathogen $B$. graminis hordei requires a living host in order to survive. As such, an infected population of barley plants is maintained at INRES and this was used as inoculum source in the study. Inoculation comprised of shaking heavily infested barley plants above 4 week old healthy test plants, cv. Leibniz (KWS, Einbeck, Germany). The plants were kept at $18 / 22^{\circ} \mathrm{C}$ (night / day) temperatures and a relative humidity (RH) of $60 \%$ in a greenhouse environment.

Sugar Beet Inoculation Protocol for Cercospora Leaf Spot disease: Cercospora beticola (necrotroph pathogen) can be stored by retaining dried infected leaves. To prepare a spore suspension the dried leaves are placed in a $100 \%$ RH humidity chamber for 24 hours. This allowed the pathogen to form spores at stromas on the leaf lesions. The prepared leaves can then be cut into small sections and added to tab water and stirred to wash the spores from the leaves, with a small amount of Polysorbate ('Tween') surfactant (source: Sigma Aldrich Gmbh, Seelze, Germany) added to the water. After the leaves and water have been sufficiently mixed (c. 10 minutes), the leaves and spore suspension are separated by filtered through muslin. The number of spores per millilitre is calculated by counting spores in a Fuchs-Rosenthal chamber under a light microscope, and the suspension is diluted to the desired concentration of $3 \times 10^{3}$ spores $/ \mathrm{ml}$. The spore suspension was sprayed on to the 6 week old test plants (cv. Pauletta, KWS Einbeck, Germany), ensuring that both sides of the plant leaves are sprayed. Subsequently incubation took place in plastic bags at $100 \%$ RH for 48 hours.

Measurements were taken daily, as per the protocol in Section III $A$., of the infected plants, alongside healthy control plants, from the point of inoculation up to 30 days after (i.e. post necrosis). Six plants per pathogen treatment were measured daily; additionally six healthy plants were measured as control plants.

\section{RESUlts}

Though this initial investigation exploited a five wavelength MSI instrument, the approach is entirely scalable to larger numbers, to accommodate multivariate model-based classification approaches and more complex SVIs. For the purposes of summarizing the findings of the MSI sensor trials, the results presented here have been limited to a subset of two SVI studies on the exemplar crops and diseases using two and three wavelength SVIs.

With respect to Fig. 4, the images represent two significant stages in the early development of powdery mildew on barley plants inoculated with $B$. graminis f. sp. hordei. Each pixel in Fig. 4B and 4C corresponds to a NDVI calculation, as per Eqn. 1 , where the NIR illumination is provided by an $830 \mathrm{~nm}$ LED and the visible red light from a $670 \mathrm{~nm}$ source. These substitutions are justified as NDVI measurements allow for this degree of tolerance, whilst having negligible effects on the readings. The reference HSI instrument was similarly configured to measure NDVI at the 830 and $670 \mathrm{~nm}$ bands. To 
aid clarity, the NDVI images, in Fig. 4, have been false-colored from blue (low values) to red (high values) using the 'jet' color map [21]

A course wire graticule was used to hold the leaves perpendicular to the camera assemblies and also act as a position index across images. Powdery mildew has been used to illustrate the sensor system operation as the NDVI can readily detect the spread of mycelia from $B$. graminis f. sp. hordei spores as they spread and obscure the leaf's surface reflectance. The corresponding results from a visual camera, the reference HSI instrument and the new MSI sensor system are shown in Fig. $4 a, 4 b$ and $4 c$, respectively. These indicate that the greater pixel density of the modified D-SLR camera (12.2 effective megapixels, $4,272 \times 2,848)$ combined with the ability to illuminate the sample with relatively high intensity light only at the SVI relevant wavelengths enabled the MSI sensor to achieve greater spatial and spectral sensitivity than the HSI instrument, without thermal or light stressing the sample.
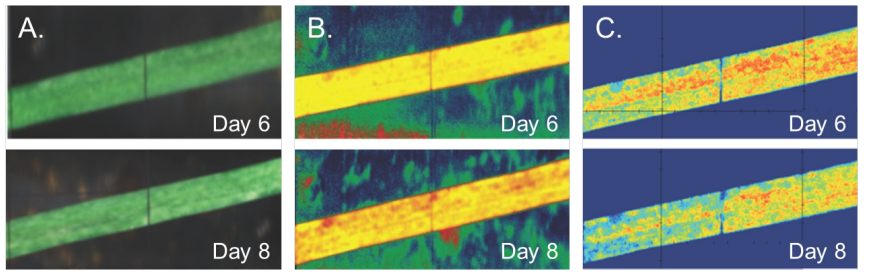

Fig. 4. Powdery mildew infected barley leaf after 6 and 8 days postinoculation: (A) Color camera image; (B) NDVI image from reference HSI instrument and; (C) NDVI image from MSI sensor system.

Fig. 5 provides results from the new MSI sensor system for Cercospora leaf spot disease in sugar beet using the CLS index (4). Similar results from the reference HSI instrument, and the definition of the CLS index, having been previously reported [16], i.e.:

$$
C L S=\frac{R_{698}-R_{570}}{R_{698}+R_{570}}-R_{734}
$$

Where: $R_{734}, R_{698}$ and $R_{570}$ are the reflected light intensities at 734, 698 and 570nm wavelengths, respectively. Due to the lack of availability, from standard suppliers, of suitable format LEDs at the $698 \mathrm{~nm}$ wavelength a $670 \mathrm{~nm}$ source was substituted in the MSI instrument and a $735 \mathrm{~nm}$ source provided the illumination for the $R_{734}$ measurements, as the required wavelength was suitably close to be within the FWHM envelope of the selected LED.

Comparison of the visual camera image (Fig. 5A), with the false-color ('jet' color map) CLS SVI image (Fig. 5B) illustrates the sensitivity of the CLS index for detecting the onset of the disease. The early symptoms on the leaf by the Cercospora leaf spot being indistinct within the visual image but clearly observable as distinct lighter colored patches in the spectrally enhanced CLS index image (3 examples circled in images). Fig. 5B was recorded by the new MSI sensor system and, again, demonstrated that the unit could provide results for the SVIs at least as detailed as that from the reference HSI instrument but with a higher spatial resolution.
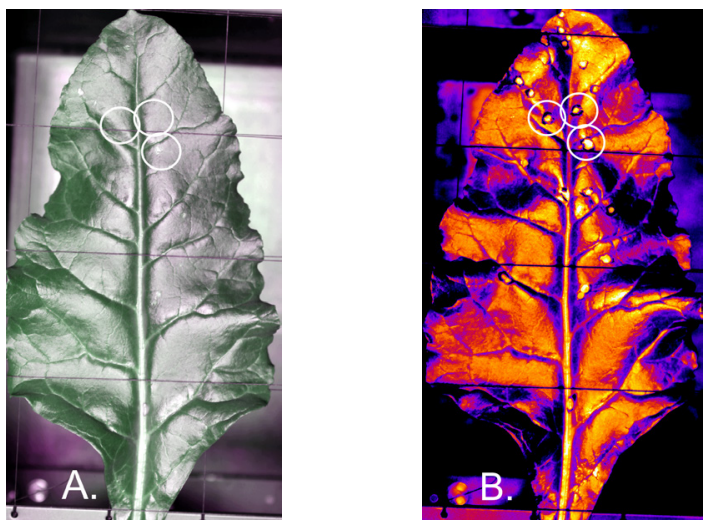

Fig. 5. Cercospora leaf spot infected sugar beet leaf: (A) Color camera image and; (B) CLS index image from MSI sensor system.

\section{CONCLUSIONS AND DISCUSSION}

The study verifies, for the first time, that multispectral sensor systems architectures, exploiting proprietary narrowband LEDs and $\mathrm{Si}$ C-MOS imaging detectors, are capable of substituting for more conventional line-scanning hyperspectral imaging systems, when operated in close proximity (c. $1-2 \mathrm{~m}$ ) from a crop canopy. This has been reported versus early symptom detection for two, economically significant, fungal pathogen caused diseases and their corresponding host crops. Two and three wavelength component SVIs have been presented, however, the extrapolation of the technique to greater numbers of wavelengths, for more complex SVIs or to generate pseudo hyperspectral images, would be a straightforward task. As a consequence, the system now offers a commercially viable route to translate crop and soil SVIs and hyperspectral models into engineered systems that maybe integrated within farm and glasshouse machinery to manage fungal diseases in crop production. Similarly, the technique also offer potential for commercial-scale localized real-time sensing of non-visual phenotypes in crop breeding programs and SVIs relevant to soil organic carbon composition, pre-harvest milling wheat protein content and intra-row weed mapping.

The next hardware engineering challenge is to develop the fixed-frame image capture techniques, as exemplified during the current laboratory trials, so that they may be reproduced from mobile, ground based, sensor platforms. For early adoption tractor-mount sensors would be the initial target as modern field machinery, enabled with: Radar ground speed sensors; Wheel rotation encoders and; Real Time Kinematic (RTK) Global Positioning Systems (GPS), capable of $\pm 20 \mathrm{~mm}$ location accuracy, provide a ready source of meta-data to enable 'stitching' of the individual wavelength image locations. This is a non-trivial task, given that field applications are currently applied at up to $17 \mathrm{~km} / \mathrm{hr}$ for broad acre crops, and may require high-speed electronics (e.g. FPGA) to be coupled with indexing of the individual images via existing locationspecific data, such as crop drilling maps or tramlines ('tractor wheel furrows'), or the inclusion of artificial VIS/NIR reflective index markers in the field. Complementary handheld sensor systems may not require neighboring images to be merged to compensate for intentional movement of the viewing position but will require image stabilization to ensure that the individual wavelength images can be overlaid with the same 
spatial reference. In both cases changing image angles will also have to be compensated for, either through optical beam steering or, more ideally, the design of angle invariant SVIs and multispectral models.

If the new MSI sensor system is to be deployed within realtime mapping and closed-loop control systems, such as for the precision application of crop protection products or nutrients, then there is also a need to rapidly identify salient features from the multispectral data. The application of linear and non-linear multivariate models to determine the most pertinent spectral components corresponding to a crop feature, using supervised and non-supervised learning techniques, is the basis of the fungal pathogen models exploited within this study and by researchers elsewhere [6-11]. Such approaches also offer the potential to swiftly compute reduced-dimensionality MSI data whilst retaining the majority of the original spectral information. The resulting 'spectrally enhanced' images then significantly reduce the computational complexity of autonomously extracting additional spatially relevant crop features. For example the spectral signature of a secretome from both benign and pathogenic fungal spores may be similar but distinct from the background spectra of the host plant's leaf. The spectral analysis alone would therefore be incapable of separating pathogenic from non-pathogenic infected leaves. However, dimensional reduction by multivariate analysis may highlight, in 'false color', otherwise indistinct patches of the secretome, such that simplified, i.e. high speed and low computationally-intensive, spatial analysis can be applied to determine potentially unique spore infiltration patterns.

Research into each of these areas is being actively pursued by the authors. Further developments of the technology to: Extend the spectral range: Incorporate fluorescence relaxation imaging and; Embed the sensors within robotic units for field, glasshouse and laboratory usage, are also underway. The exact engineering of the MSI sensor systems is highly dependent on the eventual duty, whether it is orientated towards the large commercial farming applications or for $<\mathrm{US} \$ 100$ handheld devices applied to new opportunities in livestock healthcare or crop disease control in smallholder farming or consumer products. Perhaps the largest challenge is to now identify where the sensor system may have greatest beneficial impact on sustainable food supply.

\section{ACKNOWLEDGMENTS}

The authors would like to thank the UK EPSRC for the travel funds to enable the collaborative investigation to be undertaken at INRES, University of Bonn and the EEE, University of Manchester. This work could further be carried out due to the financial support of the German Federal Ministry of Education and Research (BMBF) within the scope of the competitive grants program: Networks of excellence in agricultural and nutrition research - CROP.SENSe.net (Funding code: 0315529), subproject GS4.

\section{REFERENCES}

[1] Blackburn G.A., 2007, "Hyperspectral remote sensing of plant pigments", J Exp Bot, vol 58, pp. 844-867.
[2] Gitelson A.A., Gritz Y. and Merzylak M.N., 2003, "Relationships between leaf chlorophyll content and spectral reflectance and algorithms for non-destructive chlorophyll assessment in higher plant leaves", J Plant Physiol, vol 160, pp. 271-282.

[3] Jacquemoud S and Ustin S.L., 2001, "Leaf optical properties: A state of the art", Proc. 8th International Symposium Physical Measurements \& Signatures in Remote Sensing CNES, Aussois (France), pp. 223-232.

[4] Apan A., Held A., Phinn S. and Markley J., 2004, "Detecting sugarcane 'orange rust' disease using EO-1 Hyperion hyperspectral imagery". International Journal of Remote Sensing, vol 25, pp 489-498

[5] Rouse J.W., Haas R.H., Schell J.A. and Deering D.W., 1974. "Monitoring vegetation systems in the Great Plains with ERTS", In: Fraden S.C., Marcanti E.P. and Becker M.A. (eds), Third ERTS-1 Symposium, 10-14 Dec. 1973, NASA SP-351, Washington DC. NASA, pp. 309-317

[6] Sarlikioti, V., Driever, S.M. and Marcelis, L.F.M. 2010, "Photochemical reflectance index as a mean of monitoring early water stress", Ann. Appl. Biol., vol 157, pp 81-89

[7] Hillnhütter, C., Mahlein, A.K., Sikora, R.A. and Oerke, E.C., 2011, "Remote sensing to detect plant stress induced by Heterodera schachtii and Rhizoctonia solani in sugar beet fields", Field Crops Research, vol 122, pp 70-77

[8] Mahlein, A.K., Steiner, U., Dehne, H.W. and Oerke, E.C., 2010, "Spectral signatures of sugar beet leaves for the detection and differentiation of diseases", Precision Agric, vol 11, pp 413-431

[9] Herrmann, I., Shapira, U., Kinast, S., Karnieli, A. and Bonfil, D.J, 2013, "Ground-level hyperspectral imagery for detecting weeds in wheat fields", Precision Agric., vol 14, pp 637-659

[10] Rodionov, A., Welp, G., Damerow, L., Berg, T., Amelung, W., and Patzold, S., 2014, "Towards on-the-go field assessment of soil organic carbon using Vis-NIR diffuse reflectance spectroscopy: Developing and testing a novel tractor-driven measuring chamber", Soil \& Tillage Research, vol 145, pp 95-103

[11] Apan A., Kelly R., Phinn S., Strong W., Lester D., Butler D. and Robson A., 2006, "Predicting Grain Protein Content in Wheat Using Hyperspectral Sensing of In-season Crop Canopies and Partial Least Squares Regression", International Journal of Geoinformatics, vol 2(1), pp. 93-108

[12] Lichtenthaler H.K., 1987, "Chlorophyll fluorescence signatures of leaves during the autumnal chlorophyll breakdown", J. Plant Physiol., vol 131, pp 101-110

[13] Sahba, K., Askraba, S., and Alameh, K.E., 2006, "Non-contact laser spectroscopy for plant discrimination in terrestrial crop spraying," Opt. Express, vol. 14, pp. 12485-12493

[14] Svensgaard, J., Roitsch, T. and Christensen S., 2014, "Development of a Mobile Multispectral Imaging Platform for Precise Field Phenotyping", Agronomy, vol 4, pp 322-336

[15] Berntsen, J., Thomsen, A., Schelde, K., Hansen, O.M., Knudsen, L., Broge, N., Hougaard, H. and Hørfarter, R., 2006, "Algorithms for sensorbased redistribution of nitrogen fertilizer in winter wheat", Precision Agric., vol 7, pp 65-83

[16] Mahlein, A.K., Rumpf, T., Welke, P., Dehne, H.W., Pluemer, L., Steiner, U. and Oerke, E.C., 2013, "Development of spectral indices for detecting and identifying plant diseases", Remote Sens. Environ., vol 128, pp2130

[17] Darmont, A., 2009, "Spectral Response of Silicon Image Sensors", White Paper, April, Aphesa SPRL

[18] Sze, S.M., 1981, "Physics of Semiconductor Devices", John Wiley and Sons, N.Y.

[19] Jellison, Jr G.E. and Modine, F.A., 1982, "Optical absorption of silicon between 1.6 and $4.7 \mathrm{eV}$ at elevated temperatures", Appl. Phys. Lett- vol 41(2), pp 180-182.

[20] Mahlein A.K., Steiner U., Hillnhütter C., Dehne H.W., Oerke E.C. (2012) Hyperspectral imaging for small-scale analysis of symptoms caused by different sugar beet diseases. Plant Methods 8:3.

[21] Cox, D.J. 1988, "Using the Supercomputer to Visualize Higher Dimensions: An Artist's Contribution to Scientific Visualizing", Leanardo, vol 21(3), pp 233-24 